\title{
Promastigote Existence in Infected Lesions of Cutaneous Leishmaniasis
}

Mohammed Wael Daboul ${ }^{*}$

Daboul Medical Laboratory, Damascus University, Syria, Saudi Arabia

*Corresponding author: Mohammed Wael Daboul, Doctor in Dentistry, Daboul Medical Laboratory, Damascus University, Syria, Saudi Arabia, Tel: 0096311 3349950; E-mail: mowaeldaboul@yahoo.com

Received date: September 9, 2014; Accepted date: October 15, 2014; Published date: October 28, 2014

Copyright: ( 2014 Daboul MW. This is an open-access article distributed under the terms of the Creative Commons Attribution License, which permits unrestricted use, distribution, and reproduction in any medium, provided the original author and source are credited.

\begin{abstract}
Leishmaniasis is an endemic parasitic disease in 88 countries. It is widely distributed throughout the world, caused by vector-borne, obligate, intracellular hemoflagellates of the genus leishmania. The parasite continues its life cycle transforming to promastigote in the midgut of the sandfly vector and is transmitted to the human host in the form of promastigote through the bite of the sandfly. Other less encountered forms of transmissionare because of a laboratory accident, direct person-to-person transmission, organ transplant and blood transfusion. There is evidence that leishmaniasis may be transmitted either in utero or during the peripartum period. The promastigote form is considered the primary organism of disease transmission between the vector and the host. By not having a chance to continue its life cycle and transform into promastigote within the vector sandfly, and considering the many different routes of transmission other than the sandfly bites, it is reasonable to assume an alternative possible existence of the promastigote form of the parasite in the infected lesion of cutaneous leishmaniasis in human host. The information presented below indicates that a real transformation of amastigote to promastigote form occurs within the human host cutaneous lesion in the extracellular fluid after the macrophage membrane eruption and the amastigote release. New techniques are recommended for future studies to confirm these findings including realtime Polymerase chain reaction (PCR) and applying the immunohistochemistry techniques using novel monoclonal antibody $(\mathrm{mAb})$ against the parasite flagellate (promastigote form) cell wall component.
\end{abstract}

Keywords: Leishmaniasis; Parasitic disease; Human host; Promastigote

\section{Introduction}

Leishmaniasis is a parasitic disease widely distributed throughout the world, which is endemic in the tropical and subtropical regions of 88 countries. About 2 million cases are estimated to occur annually worldwide, most of them presenting the cutaneous or mucosal form [1]. Leishmaniasis is caused by vector-borne, obligate, intracellular hemoflagellates of the genus leishmania. The various species of leishmania are transmitted by the bite of sandfly belonging to the genus Phlebotomus. Sandflies acquire the amastigote directly from infected skin or by ingesting parasites circulating in the blood of the reservoir host. In the sandfly, the amastigote transforms into a flagellate promastigote that is infective to humans. After inoculation of the promastigote into a human host, the organism enters macrophages or histiocytes that are present in dermal tissue causing the infection where it is transformed back into amastigote form, an obligatory intracellular parasite who divides by binary fission. After multiplication, daughter amastigotes reinvade neighboring histiocytes $[2]$.

Although the parasite most commonly is transmitted through the bite of the sandfly vector, it may also be transmitted as a result of a laboratory accident, direct person-to-person transmission, organ transplant, blood transfusion and inoculation. In addition, there is evidence that it may be transmitted either in utero or during the peripartum period, while keeping in mind in all those modes of transmission the presumed missing promastigote form $[3,4]$.

Other than the sandfly bite, considering the many different routes of transmission motioned above, where the promastigote form that is primarily the source of infection to humans and the one supposed to transmit the disease, and according to the information available in literature is presumably absent from the infected lesion, while the intracellular amastigote is supposed to be the only kind to be found in human host. Given the apparent paucity of research specifically addressing this topic, the purpose of this manuscript is to shed the light from the scarce studies available on the alternative presumed existence of the flagellate promastigote form that is dismissed, in the infected lesion of cutaneous leishmaniasis in human host.

\section{Literature Review}

Studies questioning the correct assessment of the mode of spread of cutaneous leishmaniasis: So far, only sporadic reports have described cases where the cutaneous leishmania parasite has spread to the blood stream and is circulating and carried by blood leukocytes from one organ to another. One study reported that leishmania was detected in peripheral blood samples from a striking proportion of blood donors living in the Mediterranean area [5]. However, the general impression has been that these cases are typical because cutaneous leishmaniasis is usually a localized lesion of the skin so it is highly unexpected to detect the amastigote form which is the only assumed kind found in human in the peripheral blood samples among all those blood donors. Included among these unusual cases are situations in which healed scars of the Aleppo button recurred several years later. With the amastigote disappearance from the lesion towards healing [6], this places a big question mark on the real cause of recurrence. In such instances, the source of the parasite was thought to be residual organisms limited to the skin lesion without further distinct details [7]. Cruz and colleagues have reported that Leishmania spp. was detected by PCR in $32-52 \%$ of the syringes discarded by Spanish injecting drug users. That indicates that the transmission 
occurred directly from human-to-human without the sandfly bite injection of an external promastigote. Moreover, they noted shared restriction fragment length polymorphisms in roughly $20 \%$ of the samples tested, which suggests that clones of leishmania may spread through the sharing of needles [8]. That clearly reveals that the disease transmission occurred by the only presumed existed parasite in the lesion supposedly the amastigote form. This may question the circulating information that the promastigote is the one who primarily transmits the disease among different hosts.

Susceptibility to cutaneous leishmania can be greatly influenced by malnutrition, immunosuppression (e.g. HIV) and host genetic background. The advances made during the last few years in the chemotherapy of neoplasias, in the use of transplants and in the therapy of autoimmune diseases have led to the reactivation of protozoosis under these conditions, thus confirming the opportunistic and residual characters of these pathogens. Intracellular parasites are known to persist lifelong in mammalian hosts after the clinical cure of the disease, but the mechanisms of persistence are poorly understood [9]. It is uncertain whether Leishmania parasites ever disappear after clinical cure of cutaneous leishmaniasis. The results suggest that clinical cure of American Cutaneous Leishmaniasis (ACL) is rarely associated with sterile cure [10]. In experimental cutaneous leishmaniasis, live parasites have been demonstrated in various strains of mice after clinical cure by chemotherapy. It has been shown that mice experimentally infected with L. major that recover from infection still harbor infective parasites many months later [11]. In the mouse model of leishmaniasis, the persistence of even a low number of parasites in the skin after healing has been shown to maintain the host as a long-term reservoir of infection for vector sandflies. In human ACL, the persistence of Leishmania parasites after treatment and clinical cure has been demonstrated by the detection of DNA in peripheral blood and scars of patients. This persistence has also been described for other Leishmania species [10]. In most of these cases the disease recurrence is related to self-transmission due to the existence of a residual parasite without proper definition whether promastigote or amastigote form.

Studies questioning the correct assessment of the cellular processes involved in cutaneous leishmaniasis: Recently, it was demonstrated that the human neutrophils were activated by L. amazonensis infection or its lipophosphoglycan (LPG) through LTB-4 production that cooperates with degranulation of neutrophils and killing of parasites [12]. Besides, the release of neutrophil-derived extracellular DNA containing antimicrobial peptides is an important neutrophil function that contributes to parasite killing [13]. Such killing to the parasite occurs in the extracellular space which is not supposed to be the appropriate environment for the amastigote to survive, hence comes the necessity for an existence of a different form of living parasite able to survive extracellularely.

Control of Leishmania infection involves a vigorous Th1depdendent cellular immune response. Therefore, the observation of the opportunistic character of this protozoosis during the last few years was no surprise, with this parasite causing infectious disease in immunosuppressed patients, particularly those infected with HIV [14].

Leishmania and HIV coinfections have been reported in 35 out of the 88 countries in which leishmaniasis are endemic. It has been demonstrated that AIDS and leishmaniasis, can interact in a vicious cycle of mutual aggravation. With AIDS, various other opportunistic infections such as tuberculosis or systemic mycoses are found in coinfected patients, demonstrating the marked immunosuppression generated in these individuals. In that particular case, Leishmania infection may possess some similarities with chronic infectious diseases such as TB primarily by both sharing the coninfection with HIV.

In summary, in infected lesions in humans the protozoa are characterized by the following: being able to survive in the extracellular fluid, can cause the disease transmission and later recurrence, can be recovered in the peripheral blood, and are able to remain hidden silent for long time. In addition to that and most importantly, these protozoa can multiply, completely survive and transmit the disease without the sandfly involvement or engagement.

The information presented above lead us to the conclusion that the presumed parasite existed in the infected human lesion and caused the disease recurrence or transmission has more or less the same features and characteristics of the promastigote form that exists in the sand fly and transmits the disease after the bite than the obligatory intracellular amastigote.

\section{Promastigote survival and the role of glycoconjugates}

Leishmania parasites are capable to survive and to proliferate due to the protection conferred by unique glycoconjugates present on the parasites' cell surface or secreted. Most of these specialized molecules are members of a family of phosphoglycans or of glycosylinositol phospholipids. The major surface glycoconjugate of all Leishmania promastigotes is a molecule called lipophosphoglycan (LPG). LPG enables the attachment of the parasite to the sandfly midgut lectin in the epithelial cells, which enables the parasite to persist in the gut during excretion of the digested blood meal (procyclic promastigotes). During metacyclogenesis of L. major promastigotes, the LPG undergoes extensive modifications allowing the parasite to detach from the midgut and migrate towards the insect's mouthparts.

Inside the mammalian host infective (metacyclic) promastigotes display anincreased resistance to lysis induced by the complement system in the blood serum. (LPG) and secreted proteophosphoglycan (PPG) glycoconjugates play a key role in protecting the promastigote against non-specific host defense and in interacting with host serum components. The longer LPG molecules expressed on metacyclic promastigotes may contribute to their resistance to serum by preventing access of the C5b-9 membrane attack complex to the promastigote membrane. LPG has a role in protecting the parasite from digestion by lysosomal enzymes. Because LPG repeating units are highly effective in scavenging hydroxyl radicals and superoxide anions, it has been proposed that they may protect promastigotes from these toxic oxygen metabolites generated during the oxidative burst. All of that could strengthen the promastigote preference over the amastigote in the process of survival within the extracellular fluid of human host outside the infected macrophage [15].

\section{Discussion}

Determining the mode of transmission of cutaneous leishmaniasis either by the sandfly bite or by other human-to-human mode excluding the sandfly bite has an important impact in reunderstanding the parasite life cycle and declaring the role of both the vector and the host. Congenital, reactivation of leishmania after HIV infection, re-infection with leishmania among drug users sharing the needles, organ transplants and inoculation are non-vector of cutaneous leishmania disease modes of transmission. While literature tells that the promastigote develops and transforms from the 
amastigote form primarily inside the vector sandfly gut and that leishmania disease transmission occurs primarily by the promastigote form of the parasite through penetration of the skin tissues after the sandfly bite. That does not seem the case in above motioned disease transmission modes [3]. The above facts of disease transmission excluding the sandfly bite may question the vector sandfly exclusive obligatory role in the parasite life cycle through amastigote to promastigote transformation [16].

\section{Going back to the articles reviewed in order}

Having the cutaneous leishmania parasite spread to the blood stream and circulate by blood leukocytes from one organ to another is not a usual case when the amastigote form is supposed to be the one circulating in the blood. Note that the author here did not define the amastigote by name but reported it as (leishmania parasite). Being an obligatory intracellular parasite, the amastigote is usually localized inside the macrophage and does not normally spread to other organs. The report here disagrees with the basic understanding of cutaneous leishmania disease pathology.

The study reported that leishmania was detected in peripheral blood samples from a striking proportion of blood donors living in the Mediterranean area. By revealing an existence of a striking proportion that means that the vast majority of infected individuals with the disease are presenting the amastigote in their peripheral blood contrary to the disease basic scientific concept as being a localized lesion. Again note that authors referred to the parasite without naming it amastigote. The general impression has been that these cases are atypical. This is obviously due to the disagreement between the original scientific concept and the observation. Considering the promastigote existence in the host may better clarify the condition. The amastigotes though can be found outside of macrophages when they are released from them in order to infect other macrophages, that existence is a remote and transient one and for only a limited period. Amastigotes unlike promastigotes are not equipped with defense mechanisms to resist the harsh environment in the extracellular fluid. Procyclic and metacyclic promastigotes of all Leishmania species express high amounts of LPG on their surface, in contrast to amastigotes, whose LPG expression is highly downregulated (1000 old or more). Accordingly, amastigotes have limited capacity to resist the lysing function of the complement system of the body fluid.

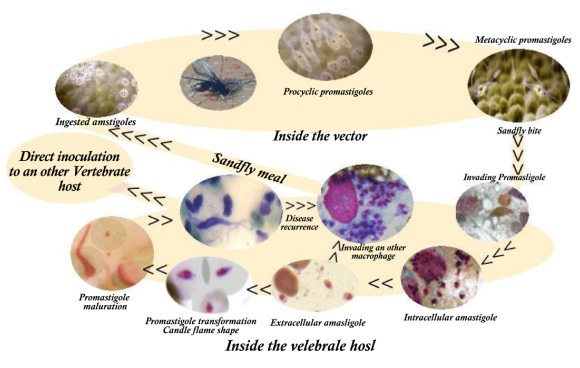

Cutaneous leishmania parasile life cycle

Figure 1: Cutaneous Leishmania parasite life cycle.

That beside other factors is a key issue in the destruction of the amastigotes and their disappearance once outside the infected macrophage. While the promastigote existence with the protecting LPG and PPG glycoconjugates against non-specific host defense prevents the interaction with host serum components and protects against the access of the complement mediated lysis through the action of C5b-9 membrane attack complex.

Non-infective procyclic promastigotes of all Leishmania species are extremely sensitive to fresh serum. L. major procyclic promastigotes rapidly activate the complement cascade via the alternative pathway, with deposition of covalently bound $\mathrm{C} 3 \mathrm{~b}$ on the parasite surface, while metacyclic promastigotes display an increased resistance to lysis [15]. It is logical to assume that the transformed promastigote form existed in the vertebrate host with its specifications and for survival reasons is more likely to be like the metacyclic one but that matter remains to be elucidated (Figure 1).

Aleppo button recurrence was reported as an unusual case due to the amastigote disappearance from the lesion towards healing. With the amastigote disappearance the recurrence must have taken place by a residual parasite reactivation supposedly in this case another transformed type.

In the case of Leishmania sp detected by PCR in $32-52 \%$ of the syringes discarded by Spanish injecting drug users, the transmission of the disease was achieved by inoculation which is atypical as being an additional but a rare type mode of transmission. This may open the question wide on the misconception of the exclusive existence of the amastigote in the mammalian host, and from there turning in to the true role of both the vector and the host.

In case of shared restriction fragment length polymorphisms in roughly $20 \%$ of the samples tested noted in those drug users sharing needles, the data in this study in particular, excludes the sandfly mode of disease transmission. This confirms the direct inoculation, raising the question of the promastigote existence within the infected tissues in the host.

The acceptance of the fact that immunosuppression (e.g. HIV) and the chemotherapy of neoplasias in the use of transplants and in the therapy of autoimmune diseases have led to the reactivation of protozoosis under these conditions. This is a true positive addition to the understanding of the disease concept revealing the opportunistic and residual characters of the pathogens and admitting that the pathogen in the host is capable by himself to reinitiate the disease. Having an opportunistic and residual characters, the parasite entitled to, is unlikely to be the amastigote form. It must be other transformed form. Opportunistic means being able to reactivate the disease conditions once the circumstances change in favor of the parasite. Such reactivation must involve the promastigote flagellate whose primary task is disease transmission or reactivation.

The neutrophil function with degranulation and DNA release taking place in the extracellular fluid is considered as a sign of the extracellular existence of the protozoa in the host; hence the reaction of the neutrophils is correspondent to that.

By having the parasite causing infectious disease the question goes why in immunosuppressed patients, particularly those infected with HIV? The answer is because leishmania infection involves a vigorous Th1-depdendent cellular immune response. The observation that incubation of a human T cell line with purified LPG induced HIV-1 LTR activity led to the suggestion that Leishmania infection may contribute to the pathogenesis of HIV infection [15]. Th1 helper is mainly affected by the HIV virus and evidently, the cellular immune response is disturbed, but how could that effect the disease reestablishment? It is due to the opportunistic character of the 
parasite, meaning its ability to reactivate itself within the tissues and produce the disease again. That concludes that a residual organism is the one that reproduces the disease and that is out of the amastigote capacity.

The residual nature of the parasite inside the host in the mouse model of leishmaniasis study together with its infectivity, illustrate an eulogistic character of the protozoa as it is capable of both transmission and reestablishment of the disease taking the role of both the amastigote and the promastigote. Putting all the above facts together, with the sandfly role exclusion, one may conclude that either the disease transmission among humans takes place through an undiscovered promastigote existence hidden somewhere in human skin lesion, or the disease transmission could be achieved by the amastigote form as being the only type of leishmania parasite presumably exists in human $[17,18]$. Still in such case, an important question arises: if it is the amastigote then how could such an obligatory intracellular parasite transmit the disease into another host? Could it be a transmission as a whole of the infected macrophage containing the infecting intracellular amastigotes together to the new host? The delicacy of the condition may not allow such transmission to take place this way. Even with this presumption, the amastigote in its form is still incapable to transmit the disease because once outside the infected cell, and being transient in a harsh aggressive environment, it cannot survive long as literature tells $[19,20]$. Being an obligatory intracellular parasite within the tissues of humankind, the amastigote form of the parasite lacks the capacity and not suited technically, functionally or physically to transmit the disease into another host unless otherwise precisely transformed into promastigote form. In order for the disease to be transmitted then, the promastigote form must exist for such transmission to take place. That concludes that some kind of real transformation of the amastigote to promastigote form must have taken place in human cutaneous infected lesion (Figure 2) [21].

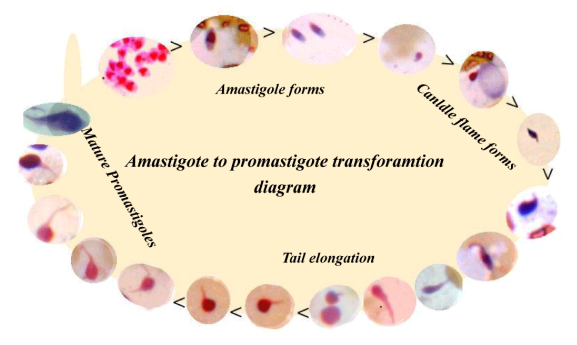

Figure 2: Amastigote to promastigote transformation.

At a later stage, while the disease itself is still in process and no complete cure yet, the disappearance of the amastigote form (LD. Bodies) from the microscopic smear with the persistence of the parasite as demonstrated by the DNA detection in the scars as declared by the American Cutaneous Leishmaniasis (ACL) study may again strongly suggest the existence of the parasite in a transformed and modified morphology that was dismissed or could not be identified by the smear conventional microscopic procedure [6].

Fibroid processes compatible with healing were found in the scars, although residual inflammatory foci were observed in some samples. This result is in agreement with systematic histopathological studies concluding that clinical cure did not always coincide with histopathological cure. Follow-up of this condition for over 15 years, the data in hand supported with many microscopic images concludes that a real transformation of amastigote to promastigote form occurred within the lesion in the extracellular fluid after the macrophage membrane eruption and the amastigote release [21-23] (Figure 3).
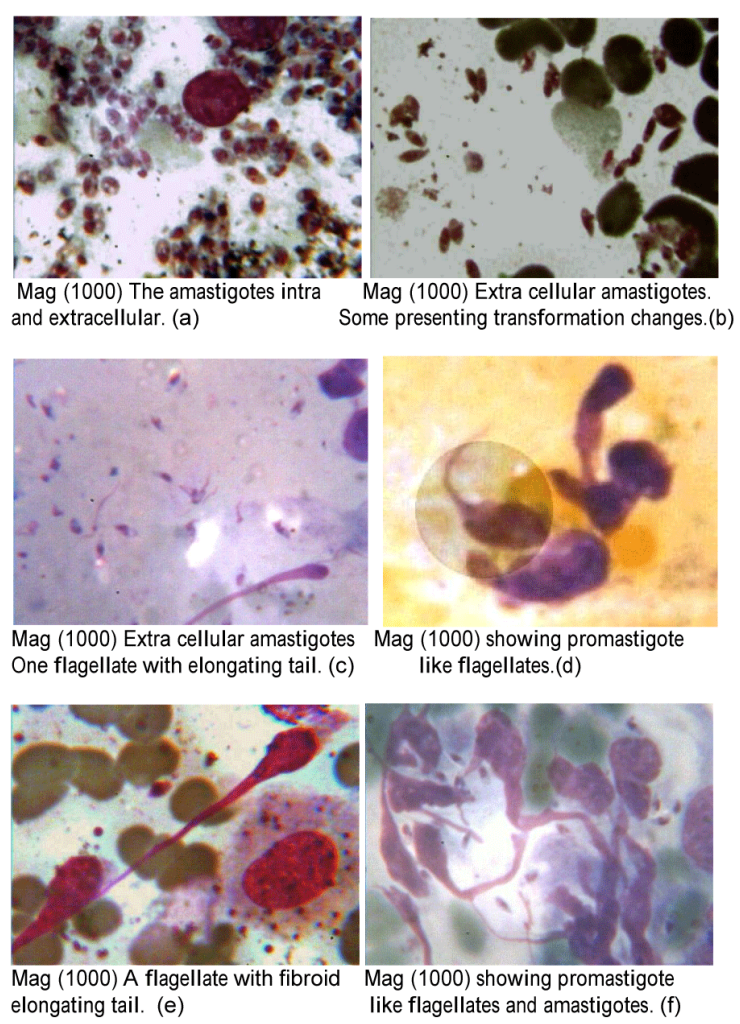

Figure 3: Images showing the transformation of the amastigote form in the extracellular fluid of human skin lesion to a flagellatelike promastigote form (Wright stain).

In his study "Wild Gorillas as a Potential Reservoir of Leishmania major J Infect Dis". Ibrahim Hamad et al. confirmed the existence of the promastigote type of the parasite in stool acquired from wild Gorillas. In his words, next fluorescence in situ hybridization was performed to visualize L. major parasites in fecal samples from the gorillas. Both promastigote and amastigote forms of the parasite were found. This work strongly suggests that wild gorillas carry pathogenic Leishmania parasites [24].

Laura Quin onez-D1'az et al. in her experimental study titled "Effect of Ambient Temperature on the Clinical Manifestations of Experimental Diffuse Cutaneous Leishmaniasis in a Rodent Mode" came with very interesting findings supporting and proving the existence of the promastigote form in the vertebrate host. She wrote, "Promastigote forms on the skin surface could preclude the detection of Leishmania because this parasite form is not expected by physicians or laboratory workers, and is not easily detected because it is embedded in squamous epithelium. In fact, doubt has recently emerged as to whether the amastigote is the only form of Leishmania present in cutaneous leishmaniasis (Daboul 2008), as was seen during a review of slides prepared from cutaneous lesions, where in $22 / 42$ 
Page 5 of 7

cases promastigote forms were observed, which is consistent with our findings. In fact, parasite forms are not easily distinguished because they are often incorporated into scales or bound to hair, where immunofluorescence is important to visualize them" [25].

Our findings and the studies presented here strongly suggest that the amastigote form at certain stage of the disease process is released from the infected macrophage into the extracellular fluid [23]. There in the extracellular fluid, a survival process with a stepwise transformation of many of the released amastigote forms into promastigote form is attained [21]. The mechanism of such transformation is similar to what happens within the gut of the sandfly (Figure 4).

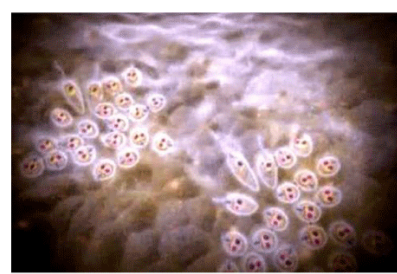

Mag (1000) The amas tigotes appearance in the midgu $t$ of the Sandfly. (a)

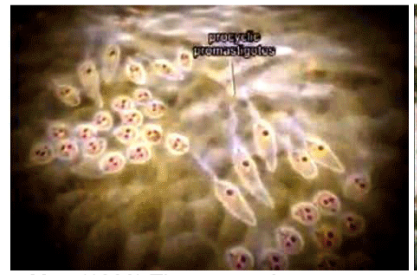

Mag (1000) The promastigote transformation within the midgut Of the sandfly (c)

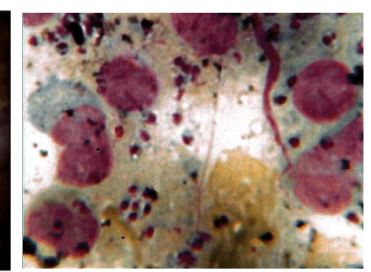

Mag (1000) The amastigote appearance in the cutaneous tissues of infected lesion from human. (b)

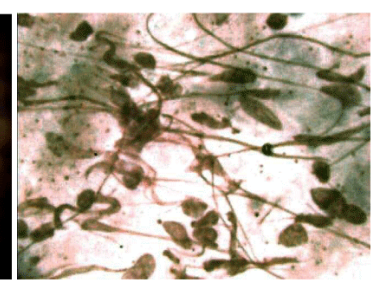

Mag (1000) The promastigote transformation in the infected lesion. in human skin (d)
Figure 4: A microscopic comparison in cytomorphology between the parasite appearance and transformation in both the mid got of the sandfly victor and the extracellular fluid of the skin infected lesion in human (The life cycle of leishmania in the insect vector" produced by Dirceu E. Teixeira, Marlene Benchimol, Juliany C. F. Rodrigues, Paulo Henrique Crepaldi, Paulo F. P. Pimenta and Wanderley de Souza).

This corroborates the persistence of an apparent infection by various Leishmania species usually associated with cutaneous leishmaniasis. This phenomenon is particularly important in view of the increasing coexistence of AIDS and leishmaniasis. It is relevant to investigate whether immunocompromised, clinically cured patients can act as reservoirs of leishmaniasis in areas of active transmission.

Table 1 declares the differences seen between traditional (e.g., sand fly) and alternative (e.g., human host) routes of transmission in cutaneous leishmaniasis.

\begin{tabular}{|l|l|}
\hline Traditional route & Alternative route \\
\hline Vector (sandfly) involvement & No vector involvement \\
\hline $\begin{array}{l}\text { Procyclic and metacyclic promastigote } \\
\text { generation }\end{array}$ & Transformed form of promastigote \\
\hline
\end{tabular}

\begin{tabular}{|l|l|}
\hline $\begin{array}{l}\text { Disease Transmission by metacyclic } \\
\text { form }\end{array}$ & Transmission with transformed form \\
\hline $\begin{array}{l}\text { Amastigote ingestion from the host into } \\
\text { the sandfly vector }\end{array}$ & $\begin{array}{l}\text { Amastigote stepwise transformation to } \\
\text { promastigote in the extracellular fluid }\end{array}$ \\
\hline $\begin{array}{l}\text { Metacyclic promastigote skin } \\
\text { penetration }\end{array}$ & Direct inoculation or latent reactivation \\
\hline $\begin{array}{l}\text { Different parasite strains may be } \\
\text { involved }\end{array}$ & $\begin{array}{l}\text { One strain of parasites is mainly } \\
\text { involved }\end{array}$ \\
\hline
\end{tabular}

Table 1: Differences between traditional (e.g., sand fly) and alternative (e.g., human host) routes of transmission in cutaneous leishmaniasis.

It has been noticed that leishmania parasite infection with its chronic sequel characterized at the end stage with scar formation and no full recovery of the cutaneous tissues, in many aspects may share similarities with Mycobacterium tuberculosis infection. Both can survive for a long time in the body of a healthy host, causing no symptoms after primary infection. Years later, M. tuberculosis within the old foci can be reactivated in some TB carriers, leading to symptoms of post-primary tuberculosis. While in case of cutaneous leishmaniasis, leishmania parasites present in the skin scar and by the aid of sandfly bite (or by the other modes of transmission including immuno-suppression effects) will lead to reinfection or recurrence and resuming the parasite life cycle.

During the asymptomatic period, M. tuberculosis is believed to survive in old foci of infection, while the parasite in case of cutaneous leishmaniasis embalms itself within the scar tissues in the skin [21]. Yet these foci in case of TB are uniformly negative by culture and acidfast staining, which are the conventional methods to detect mycobacteria. Similarly, the Leishmen-Donovan method, which is the conventional microscopic method to detect the parasite in case of cutaneous leishmaniasis, becomes at the later stages of the disease process negative for the LD bodies [6].

The precise location and amount of M. tuberculosis in these foci in case of primary $\mathrm{TB}$, or the leishmania parasite persistence in case of the terminating stage of cutaneous leishmaniasis therefore, are not well understood. In cutaneous leishmaniasis, one explanation for the reason behind this negativity in identifying the parasite by microscope is that the microscopic method has its limitation and detects only the LD Bodies within the macrophage, which is the principle of such microscopic method for diagnosing cutaneous leishmaniasis. Since the time of Leishmen-Donovan and over hundred years ago, a concrete perception which later converted into a form of adoption and never questioned or reviewed again was accepted revealing that only the intracellular amastigote exists in human host. While in-fact, certain amounts of L. parasites that remain have already lost their normal morphology (the amastigote form) and transformed into a flagellate like structure in the histological sections of the old lesions, but missed by the classical microscopic method [23]. Otherwise, how could we simply explain the disappearance of the amastigote form together with the infected macrophages in many reported cases at a later stage of the disease while the disease continues to be in process for long time to come as such a disease process with the absence of an infecting cause? Towards healing, leishmania lesions seldom contain amastigote form; they contain few transformed parasites. 


\section{Conclusion}

Here we report that in case of cutaneous leishmaniasis with the scar formation, a mild granulomatous lesion with fibroid structure is a well-known characteristic of the disease progress towards healing. This fibroid structure in the lesion is characterized by the existence of the promastigote parasite flagellate embedded in many different shapes and morphologies [23]. This promotes that the end case scar lesion is most likely to be positive for the flagellate parasite genome if tested by the different other lab diagnostic procedures. New techniques are recommended for future studies confirming the existence of the flagellate including checking specifically for the promastigote form of the parasite during the time of the amastigote disappearance from the microscopic smear at a later stage of the disease process. This can be done by performing real-time Polymerase Chain Reaction (PCR) which is considered a highly sensitive and specific technique for detection of the of leishmania parasite in the scar tissues of old cutaneous lesions. It may also be possible to perform the immunohistochemistry techniques using a novel monoclonal antibody $(\mathrm{mAb})$ against the parasite (promastigote form) cell wall component [26]. That requires in the future a thorough follow-up with the application of both techniques.

It is important to note that the new findings presented have a strong impact on the clinical side. The implications of such findings on the disease diagnosis are valuable. Considering the promastigote existence in the conventional smear method, this will add a lot to the method indices, reaching almost $100 \%$ with the method sensitivity and specificity and making the method one of the most valuable in diagnosing leishmaniasis. Prevention is another aspect that these findings effect. The discovery of the residual existence of the parasite for long in the host and its role in disease recurrence should direct the authority's attention to take measures for prevention especially with its ability to cause both the recurrence and the transmission [27-30]. Our findings have also their impact on the disease treatment. Unfortunately, the findings tell, to date, and with the repeated disease recurrence in many cases after long time of treatment, that most of the drugs in-use is not able yet to provide a permanent cure for the disease and more studies in that direction to achieve curable drugs are highly appreciated. The risk of reactivation of latent leishmaniasis from these skin scar old fibroid lesions should always be considered especially with the sand fly existence in the endemic arias.

\section{Acknowledgement}

My ample thanks to Dr. Lamis J. Maalouf for her critical reading, contribution and the great effort she put in this work.

\section{References}

1. Machado CM, Martins TC, Colturato I, Leite MS, Simione AJ, et al. (2009) Epidemiology of neglected tropical diseases in transplant recipients. Review of the literature and experience of a Brazilian HSCT center. Rev Inst Med Trop Sao Paulo 51: 309-324.

2. Leite S, Simione AJ, de Souza MP, Mauad MA, Colturato VR (2009) Epidemiology of neglected tropical diseases in transplant recipients. Rev Inst Med trop.51: 6 .

3. Abass K, Saad H, Abd-Elsayed AA (2009) The first case of isolated facial cutanenous leishmaniasis in a Down syndrome infant: a case report and review of the literature. Cases J 2: 13.

4. Vidyashankar C, Agrawal R (2006) Leishmaniasis. E-Medicine Specialties.
5. World Health Organization. Leishmaniasis. Disease information. TDR diseases.

6. le Fichoux Y, Quaranta JF, Aufeuvre JP, Lelievre A, Marty P, et al. (1999) Occurrence of Leishmania infantum parasitemia in asymptomatic blood donors living in an area of endemicity in southern France. J Clin Microbiol 37: 1953-1957.

7. Hepburn N (2006) Cutaneous leishmaniasis. Clin Exp Dermatol 25: 363-3705.

8. Nakkash-Chmaisse H, Makki R, Nahhas G, Knio K, Nuwayri-Salti N (2011) Detection of Leishmania parasites in the blood of patients with isolated cutaneous leishmaniasis. Int J Infect Dis 15: e491-494.

9. Nuha Nuwayri-Salti (2011) Detection of Leishmania parasites in the blood of patients with isolated cutaneous leishmaniasis. International Journal of Infectious Diseases 15: e491-e494.

10. Pineda JA, Martín-Sánchez J, Macías J, Morillas F (2002) Leishmania spp infection in injecting drug users. Lancet 360: 950-951.

11. Centers for Disease Control and Prevention (CDC) (2002) Outbreak of listeriosis--northeastern United States, 2002. MMWR Morb Mortal Wkly Rep 51: 950-951.

12. Bogdan C, Donhauser N, Döring R, Röllinghoff M, Diefenbach A, et al. (2000) Fibroblasts as host cells in latent leishmaniosis. J Exp Med 191: 2121-2130.

13. Mendonça MG, de Brito MEF, Rodrigues EHG, Bandeira V, Jardim ML et al. (2004) Persistence of Leishmania Parasites in Scars after Clinical Cure of American Cutaneous Leishmaniasis: Is There a Sterile Cure? J Infect Dis 189: 1018-1023.

14. Aebischer T, Moody SF, Handman E (1993) Persistence of virulent Leishmania major in murine cutaneous leishmaniasis: a possible hazard for the host. Infect Immun 61: 220-226.

15. Tavares NM, Arajo -Santos T, Afonso L, Nogueira PM, Lopes UG, et al. (2014). Understanding the mechanisms controlling Leishmania amazonensis infection in vitro: the role of LTB4 derived from human neutrophils. J. Infect. Dis.

16. Abi Abdallah DS, Denkers EY (2012) Neutrophils cast extracellular traps in response to protozoan parasites. Front Immunol 3: 382.

17. Uzonna JE, Joyce KL, Scott P (2004) Low dose Leishmania major promotes a transient $\mathrm{T}$ helper cell type 2 response that is down-regulated by interferon gamma-producing CD8+ T cells. J Exp Med 199: 1559-1566.

18. Descoteaux A, Turco SJ (1999) Glycoconjugates in Leishmania infectivity. Biochim Biophys Acta 1455: 341-352.

19. Parasitism \& Symbiosis. Leishmania. 177-345A.

20. Sundar S, Rai M Laboratory diagnosis of visceral leishmaniasis. KalaAzar.

21. Beena KR, Ramesh V, Mukherjee A (2003) Identification of parasite antigen, correlation of parasite density and inflammation in skin lesions of post kala-azar dermal leishmaniasis. J Cutan Pathol 30: 616-620.

22. Vidyashankar C, Agrawal R. Leishmaniasis. E-Medicine Specialties. Available at: www.emedicine.com/ped/topic1292.htm. Last Updated: February 27, 2006.

23. Cascio A, Calattini S, Colomba C, Scalamogna C, Galazzi M, et al. (2002) Polymerase chain reaction in the diagnosis and prognosis of Mediterranean visceral leishmaniasis in immunocompetent children. Pediatrics 109: E27.

24. Daboul MW (2008) Is the Amastigote Form the Only Form Found in Humans Infected With Cutaneous Leishmania? LABMEDICINE D 39.

25. Da'aboul MW (2009) [Cutaneous leishmaniasis in Damascus]. East Mediterr Health J 15: 1084-1097.

26. Daboul MW (2012) Cutaneous Leishmaniasis- A New Concept. LAP LAMBERT.

27. Hamad I, Forestier CL1, Peeters M2, Delaporte E2, Raoult D1, et al. (2014) Wild Gorillas as a Potential Reservoir of Leishmania major. J Infect Dis.

28. Quiñonez-Díaz L, Mancilla-Ramírez J, Avila-García M, Ortiz-Avalos J, Berron A, et al. (2012) Effect of ambient temperature on the clinical 
Citation: Daboul MW (2014) Promastigote Existence in Infected Lesions of Cutaneous Leishmaniasis. J Gen Practice 2: 183. doi: 10.4172/2329-9126.1000183

Page 7 of 7

manifestations of experimental diffuse cutaneous leishmaniasis in a rodent model. Vector Borne Zoonotic Dis 12: 851-860.

29. Mary Ann Liebert (2012) Effect of Ambient Temperature on the Clinical Manifestations of Experimental Diffuse Cutaneous Leishmaniasis in a Rodent Mode. VECTOR-BORNE AND ZOONOTIC DISEASES 12.
30. Chamakh-Ayari R (2014) In Vitro Evaluation of a Soluble Leishmania Promastigote Surface Antigen as a Potential Vaccine Candidate against Human Leishmaniasis. PLOS. 